\title{
Right ventricular dysfunction predict limited exercise capacity in heart failure with reduced ejection fraction
}

\author{
Pranvera Ibrahimi ${ }^{1 *}$, Gani Bajraktari', Afrim Poniku², Violeta Hysenaj', Artan Ahmeti', \\ Fisnik Jashari', Edmond Haliti' ${ }^{2}$, Michael Y Henein \\ ${ }^{1}$ Umeå University, Umeå, Sweden \\ 'University Clinical Centre of Kosova, Prishtina, Kosovo
}

Background and Aim: Compromised exercise capacity is the main symptom in patients with heart failure (HF) and reduced left ventricular (LV) ejection fraction (EF). Six-minute walk test (6-MWT) is popular for objective assessment of exercise capacity in these patients but is confined to heart centres. The aim of this study was to prospectively examine functional parameters that predict 6-MWT in patients with $\mathrm{HF}$ and reduced LVEF.

Methods: In $111 \mathrm{HF}$ patients (mean age $60 \pm 12$ years, 56\% male), a 6-MWT and an echo-Doppler study were performed on the same day. In addition to conventional ventricular function measurements, global LV dyssynchrony was indirectly assessed by total isovolumic time - t-IVT [in s/min; calculated as: 60 - (total ejection time - total filling time)], and Tei index (t-IVT/ejection time). Also, LV and right ventricular function were assessed by mitral and tricuspid annular plane systolic excursion (MAPSE and TAPSE, respectively). Based on the 6-MWT distance, patients were divided into: Group I: $300 \mathrm{~m}$ and Group II: >300 m.

Results: The 6-MWT distance correlated with t-IVT and Tei index ( $r=-0.37, p<0.001$, for both), lateral and septal e' velocities ( $r=0.41, p<0.001$, and $r=0.46, p<0.001$, respectively), E/e' ratio $(r=-0.37, p<0.001)$ and TAPSE $(r=0.45, p<0.001)$, but not with the other clinical or echo parameters. Group I patients had longer t-IVT, lower E/e'ratio, TAPSE and lateral $e^{\prime}(p<0.001$ for all) compared with Group II. In multivariate analysis, TAPSE [0.076 (0.017-0.335), $p=0.001], E / e^{\prime}[1.165$ (1.017-1.334), $\quad p=0.027]$, t-IVT [1.178 (1.014-1.370), $\mathrm{p}=0.033$ ] independently predicted poor 6-MWT performance $(<300 \mathrm{~m})$. Sensitivity and specificity for TAPSE $1.9 \mathrm{~cm}$ were $66 \%$ and $77 \%$, (AUC 0.78, p<0.001); E/e' 10.7 were $66 \%$ and $62 \%$ (AUC 0.67, p=0.002) and t-IVT $13 \mathrm{~s} / \mathrm{min}$ were $64 \%$ and $60 \%$ (AUC 0.68, $\mathrm{p}=0.002$ ) in predicting poor 6-MWT. Combined TAPSE and E/e' had a sensitivity of $68 \%$ but specificity of $92 \%$ in predicting 6-MWT. Respective values for combined TAPSE and t-IVT were $71 \%$ and $85 \%$.
Conclusion: In patients with HF, the limited exercise capacity assessed by 6-MWT, is multifactorial being related to severity of right ventricular systolic dysfunction as well as raised LV filling pressures and global dyssynchrony.

KEYWORDS: six-minute walk test, Doppler echocardiography, right ventricular function, heart failure, exercise capacity.

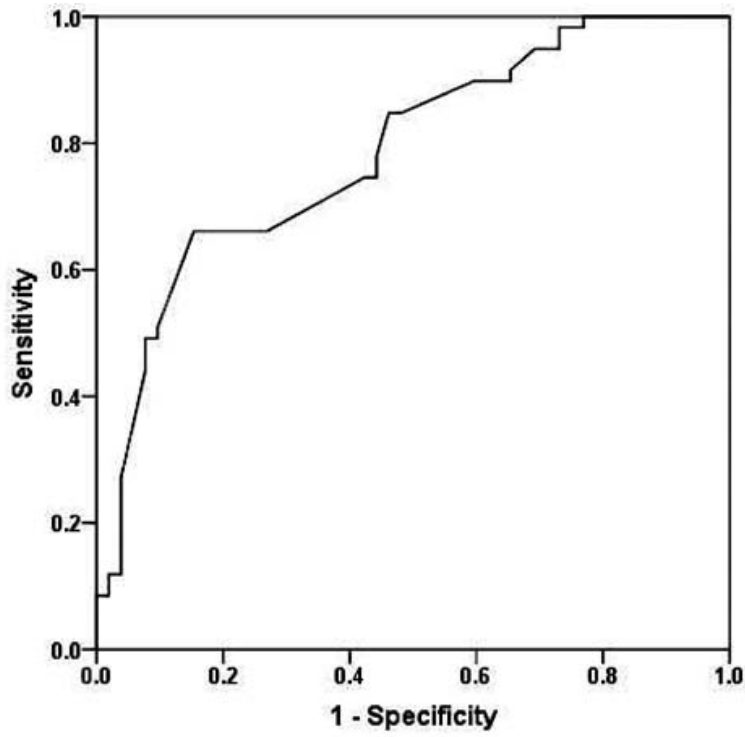

Figure 1. ROC curve of TAPSE in predicting limited exercise capacity in HF patients.

\section{Received: $20^{\text {th }}$ Mar 2013}

*Address for correspondence: Department of Public Health and Clinical Medicine, and Heart Centre, Umea University, SE-901 87 Umea, Sweden. Phone: +46-90-785 2652

E-mail: pranvera.ibrahimi@medicin.umu.se

\section{Literature}

1. Gardin JM, Leifer ES, Fleg JL, Whellan D, Kokkinos P, Leblanc MH, et al; HF-ACTION Investigators. Relationship of Doppler-Echocardiographic left ventricular diastolic function to exercise performance in systolic heart failure: the HF-ACTION study. Am Heart J. 2009;158(4 Suppl):S45-52.

2. Ghio S, Temporelli PL, Klersy C, Simioniuc A, Girardi B, Scelsi L, et al. Prognostic relevance of a non-invasive evaluation of right ventricular function and pulmonary artery pressure in patients with chronic heart failure. Eur J Heart Fail. 2013;15(4):408-14.

3. Bajraktari G, Elezi S, Berisha V, Lindqvist P, Rexhepaj N, Henein MY. Left ventricular asynchrony and raised filling pressure predict limited exercise performance assessed by 6 minute walk test. Int J Cardiol. 2011;146(3):385-9

4. D’Andrea A, Gravino R, Riegler L, Salerno G, Scarafile R, Romano M, et al. Right ventricular ejection fraction and left ventricular dyssynchrony by $3 \mathrm{D}$ echo correlate with functional impairment in patients with dilated cardiomyopathy. J Card Fail. 2011;17(4):309-17. 